\title{
FEASIBILITY OF PROCESS INTENSIFICATION OF WATER-GAS SHIFT REACTION USING A MICROREACTOR WITH INTEGRATED COOLING
}

\author{
Thomas E. Tolley, Brian M. Fronk* \\ School of Mechanical, Industrial, and Manufacturing Engineering \\ Oregon State University \\ Corvallis, OR, 97331
}

\begin{abstract}
Hydrogen is an increasingly attractive low-carbon energy carrier for a variety of stationary and mobile applications. The water-gas shift (WGS) reaction is a key processing step used for large-scale hydrogen production via the steam methane reforming process. However, the thermodynamics and kinetics of the reaction are such that standard two-stage adiabatic reactors used in these systems are large, increasing catalyst volume and cost. To intensify the process and realize the economical distributed production of hydrogen, diabatic WGS microreactors with integrated cooling directly regulate the reaction temperature via integrated coolant channels to promote higher conversion within a smaller reactor volume. This study investigates the conversion efficiency of a single WGS microchannel operating under such cooling conditions. A COMSOL Multiphysics model is developed and validated with isothermal experimental data from the literature. The model is then used to evaluate improvements in conversion efficiency when the reaction is cooled via a specified wall temperature profile. Finally, the model is modified to include cooling channels with a secondary fluid that can practically achieve a similar conversion profile as the specified wall temperature profile previously applied. Initial results show that reactor conversion can be significantly increased by the inclusion of appropriate cooling and that there is a potential for the recovery of energy from the reaction stream that can be used for other applications within the overall process.
\end{abstract}

KEY WORDS: hydrogen, water-gas shift, process intensification, microreactor, energy recovery

\section{INTRODUCTION}

The world's progression toward the hydrogen economy is currently largely facilitated by the hydrogen production capacity of the steam methane reforming process. As of $2018,48 \%$ of worldwide hydrogen was produced via the steam methane reforming process [1], making it the single largest industrial source of hydrogen. The water-gas shift (WGS) reaction is a key processing step within the steam methane reforming process that converts carbon monoxide and water vapor into carbon dioxide and hydrogen gas according to the following chemical equation:

$$
\mathrm{CO}+\mathrm{H}_{2} \mathrm{O} \rightleftharpoons \mathrm{CO}_{2}+\mathrm{H}_{2}
$$

*Corresponding Brian M. Fronk: brian.fronk@oregonstate.edu; phone: 541.737.3952 
The WGS reaction is a mildly exothermic $\left(\Delta H^{\circ}=-41.1 \mathrm{~kJ} / \mathrm{mol}\right)$, reversible reaction. As a result, the reaction is kinetically favorable at higher temperatures, but thermodynamically favorable at lower temperatures [2]. This can be directly seen by examining an expression for the equilibrium constant for the WGS reaction, which decreases substantially as reaction temperature increases [3]:

$$
K_{e q}=\exp \left(\frac{4577.8}{T}-4.33\right)
$$

Compared to other industrial reactions, the WGS reaction is relatively slow, often making WGS reactors the largest components within a given chemical system [4]. As a result, achieving the desired product yield from the WGS reaction within a reasonable reactor volume becomes a matter of effectively managing the temperature of the reaction to balance the possible equilibrium state and reaction rate. In industry, this is commonly done by splitting the WGS reaction into separate adiabatic high temperature shift (HTS) and low temperature shift (LTS) packed bed reactors [5]. The HTS reactor achieves the bulk of the reaction conversion in a lower reactor volume due to the faster reaction kinetics and the LTS reactor is then used to further increase hydrogen production due to the higher conversions possible at lower temperatures.

In the chemical industry broadly, there has been significant interest in process intensification, or the reduction of chemical plant size with commensurate increase in efficiency. As part of process intensification efforts focused on the WGS reaction, microreactors have been proposed as an alternative reactor style to packed bed reactors that can reduce the volume of such units as a result of their superior mass transfer and heat transfer characteristics [6]. Microreactors allow for more direct control over the reaction temperature via integrated coolant channels, leading to opportunities for enhanced hydrogen production and energy recovery from the reaction stream. These benefits have led to a number of groups investigating their use for the WGS reaction. Bac et al. [7] simulated the effects of integrated cooling on the operation of WGS microreactors and noted that such reactors can maintain nearly isothermal reaction temperatures, increasing reactor conversion by more than $10 \%$ compared to adiabatic reactors. Romero and Wilhite [8] investigated the effects of imposing temperature profiles on the reaction stream within WGS microreactors and found that decreasing temperature profiles were able to enhance reactor conversion beyond what is possible for isothermal reactors, with convex temperature profiles achieving the highest reactor $\mathrm{CO}$ conversion and linear temperature profiles being the most volume-efficient temperature profile. TeGrotenhuis et al. [9] more systematically evaluated the effects of temperature on the WGS reaction and developed an optimal temperature profile for a WGS plug flow reactor, which minimizes the reactor length to achieve a certain conversion. Their results indicate that rapid cooling near the reactor inlet, where the exothermic reaction quickly generates heat, significantly enhances $\mathrm{CO}$ conversion and that the bulk of the reactor conversion is achieved within the first third of the reactor.

In this study, a two-dimensional (2-D) model considering the reaction kinetics and transport phenomena of a WGS reactor is developed and validated using experimental data found in the literature. Once validated, the model is used to characterize improvements to reactor $\mathrm{CO}$ conversion while operating under an optimal temperature profile. To expand upon the work of previous authors, this study specifically develops an optimal temperature profile for a 2-D WGS reactor model as opposed to a plug flow reactor model and separately evaluates reactor performance in terms of the potential for energy recovery from the reaction stream via integrated coolant channels, suggesting it as another useful measure of WGS reactor performance.

\section{MODEL DEVELOPMENT}

This section outlines the modeling approach taken in this study. The first stage of this effort focused on the development of a 2-D, isothermal model of a single WGS microchannel and validating its accuracy using experimental data found in the literature. Then, the validated model was used to determine an optimal, imposed wall temperature profile for the WGS reaction for a given inlet condition. Finally, based on these optimal wall temperature profile results, the model was modified to include a coolant channel with a secondary fluid and 


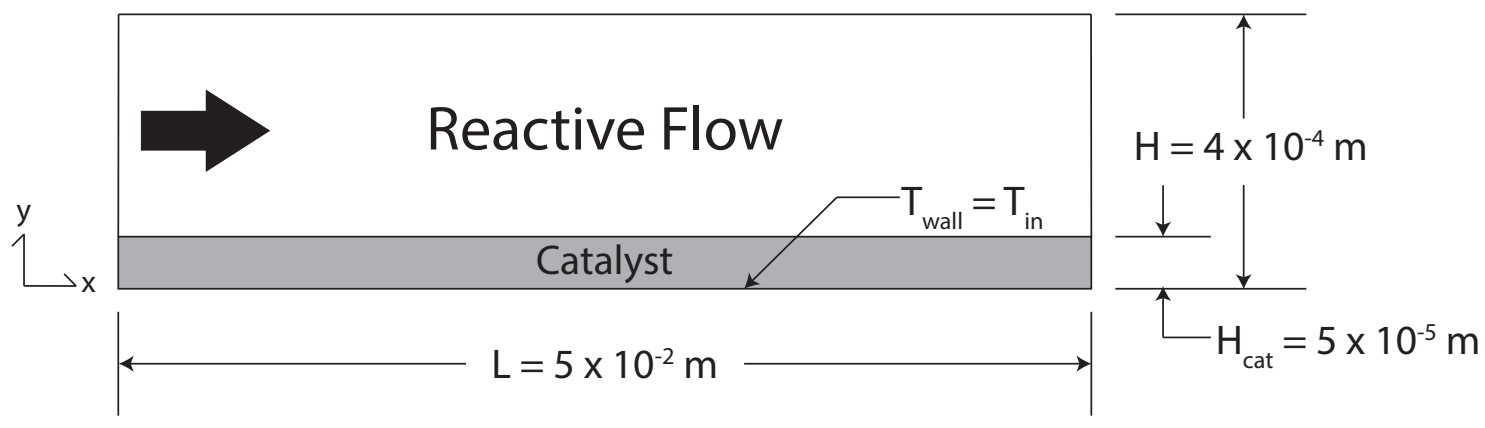

Fig. 1 2-D geometry of microchannel Model

used to develop practical methods of achieving similar temperature and conversion profiles to the optimal profile.

\subsection{Isothermal Reactor Model}

The 2-D isothermal reactor model developed in the first stage of this study emulates a single reaction microchannel operating at the experimental conditions used by Germani and Schuurman [10] in their characterization of a $\mathrm{Pt} / \mathrm{CeO}_{2} / \mathrm{Al}_{2} \mathrm{O}_{3}$ WGS catalyst. The experiment conducted by Germani and Schuurman used a reactor consisting of six microstructured, stainless steel platelets stacked on each other and sealed in an outer housing. Each platelet was $50 \mathrm{~mm} \times 50 \mathrm{~mm} \times 1 \mathrm{~mm}$ and had a total of 49 microchannels etched into it, resulting in a total of 294 microchannels being used in the reactor. Each microchannel was $0.4 \mathrm{~mm}$ deep, 0.6 $\mathrm{mm}$ wide, and spanned the entire $50 \mathrm{~mm}$ length of the plate. Each microchannel was washcoated with catalyst such that a single layer would deposit on its bottom surface. On average, $2.16 \mathrm{mg}$ of catalyst deposited in each microchannel. The catalyst density was characterized as $\rho_{\text {cat }}=1450 \mathrm{~kg} / \mathrm{m}^{3}$ during the experiment, indicating an average catalyst layer thickness of approximately $0.05 \mathrm{~mm}$ in each channel. The 2-D geometry of the microchannel used in the reactor model is shown in Figure 1.

The reaction stream fed into the reactor in the experiments conducted by Germani and Schuurman was at a pressure of 1 bar and had a composition of $10 \% \mathrm{CO}, 20 \% \mathrm{H}_{2} \mathrm{O}, 10 \% \mathrm{CO}_{2}, 30 \% \mathrm{H}_{2}$, and $30 \% \mathrm{Ar}$ on a molar basis. The feed rate of the reaction stream was fixed at $100 \mathrm{Nml} / \mathrm{min}$. In the model developed, it is assumed that there is negligible flow maldistribution and that the reaction stream splits uniformly amongst all of the microchannels in the reactor. During the experiment, the inlet temperature of the reaction stream was varied from $200{ }^{\circ} \mathrm{C}$ to $340{ }^{\circ} \mathrm{C}$. Cartridge heaters in the reactor were used to maintain nearly isothermal conditions within the reactor for each inlet temperature used.

The model developed here accounted for momentum transfer, mass transfer, and heat transfer in both the open channel and porous catalyst phases of a single reaction channel and was numerically evaluated using the experimental conditions described above in COMSOL Multiphysics. A constant wall temperature boundary condition equal to the reaction inlet temperature was applied, as shown in Figure 1, to maintain isothermal conditions within the model. The Navier-Stokes equations and Brinkman equations were used to model the momentum transfer in the open channel and porous catalyst, respectively. The varying density $\left(\rho_{m}\right)$ and viscosity $\left(\mu_{m}\right)$ of the reaction stream mixture were evaluated and used in both equations. The subscript " $m$ " is used in these equations and the other transport equations to denote these mixture properties.

$$
\begin{gathered}
\rho_{m}(\mathbf{u} \cdot \nabla) \mathbf{u}=-\nabla P+\nabla \cdot\left(\mu_{m}\left(\nabla \mathbf{u}+(\nabla \mathbf{u})^{T}\right)-\frac{2}{3} \mu_{m}(\nabla \cdot \mathbf{u})\right) \\
\frac{1}{\epsilon} \rho_{m}(\mathbf{u} \cdot \nabla) \mathbf{u} \frac{1}{\epsilon}=-\nabla P+\nabla \cdot\left(\frac{\mu_{m}}{\epsilon}\left(\nabla \mathbf{u}+(\nabla \mathbf{u})^{T}\right)-\frac{2}{3} \frac{\mu_{m}}{\epsilon}(\nabla \cdot \mathbf{u})\right)-\frac{\mu_{m}}{\kappa} \mathbf{u}
\end{gathered}
$$


The mass conservation equations applied to the open channel and porous catalyst phases for each species are nearly identical. However, the occurrence of the WGS reaction was only considered within the porous catalyst phase of the reactor. The presence of the reaction in the porous catalyst was accounted for by the inclusion of consumption and generation terms in the mass conservation equations for the reactants and products, respectively. The diffusion coefficients in the equations also differed, as gas phase diffusion coefficients for each chemical species were used in the open channel $\left(D_{i-m}\right)$ and effective porous diffusion coefficients for each chemical species were used in the porous catalyst phase $\left(D_{e, i-m}\right)$. The diffusion coefficients of single chemical species into the rest of the reaction mixture were evaluated using a mixture-averaged approach and the presence of Knudsen diffusion in the porous catalyst layer was neglected.

$$
\begin{gathered}
\nabla \cdot\left(D_{i-m} \nabla \bar{c}_{i}\right)-\nabla \cdot\left(\mathbf{u} \bar{c}_{i}\right)=0 \\
\nabla \cdot\left(D_{e, i-m} \nabla \bar{c}_{i}\right)-\nabla \cdot\left(\mathbf{u} \bar{c}_{i}\right)+r_{i}=0
\end{gathered}
$$

The energy conservation equations applied to the open channel and porous catalyst phases are also nearly identical. Heat generation due to the chemical reaction taking place was only considered in the porous catalyst phase and was accounted for by the inclusion of a heat generation term. The varying specific heat $\left(c_{p, m}\right)$ and thermal conductivity $\left(k_{m}\right)$ of the reaction stream mixture were evaluated and used in the open channel equation. The thermal properties in the porous catalyst equation were taken as volume averages of the gas phase and solid phase thermal properties $\left(c_{p, e, m}, k_{e, m}\right)$.

$$
\begin{gathered}
\nabla \cdot\left(k_{m} \nabla T\right)-\nabla \cdot\left(\rho_{m} c_{p, m} \mathbf{u} T\right)=0 \\
\nabla \cdot\left(k_{e, m} \nabla T\right)-\nabla \cdot\left(\rho_{m} c_{p, e, m} \mathbf{u} T\right)+\left(-\Delta H_{W G S}\right)\left(r_{W G S}\right)\left(\rho_{c a t}\right)=0
\end{gathered}
$$

The WGS equilibrium expression given in Equation 2 was used to evaluate the equilibrium state of the reaction at a given temperature and the reaction rate was predicted using the Langmuir-Hinshelwood expression developed by Germani and Schuurman [10] for the catalyst considered:

$$
r_{W G S}=\frac{k_{r d s} K_{C O} K_{\mathrm{H}_{2} \mathrm{O}} P_{C O} P_{\mathrm{H}_{2} \mathrm{O}}\left(1-\frac{P_{\mathrm{CO}_{2} P_{\mathrm{H}_{2}}}}{K_{e q} P_{C O} P_{\mathrm{H}_{2} \mathrm{O}}}\right)}{\left(1+K_{C O} P_{C O}+\sqrt{K_{\mathrm{H}_{2}} P_{\mathrm{H}_{2}}}\right)^{2}\left(1+\sqrt{K_{\mathrm{H}_{2} \mathrm{O}} P_{\mathrm{H}_{2} \mathrm{O}}}+K_{\mathrm{CO}_{2}} P_{C O_{2}}\right)}
$$

\subsection{Prescribed Optimal Temperature Reactor Model}

The optimal temperature profile for a WGS reactor is found by setting the partial derivative of the reaction rate with respect to temperature equal to zero [9]. In terms of a reactant $A$, this equation can be written as:

$$
\frac{\partial r_{A}\left(X_{A}, T_{\max }, \bar{c}_{i, 0}\right)}{\partial T}=0
$$

Equation 10 is a function of the inlet composition and temperature. Unique optimal temperature profiles exist for each inlet condition. Using this equation, the maximum possible $\mathrm{CO}$ conversion at each reaction rate and the corresponding temperature can be determined, as shown in Figure 2. This locus of maximum conversion points represents the reaction progression that achieves the maximum $\mathrm{CO}$ conversion in the lowest possible reactor volume.

To evaluate reactor performance while following this optimal temperature profile, the isothermal reaction channel model was modified such that it operated isothermally near the inlet of the reactor until it approached 


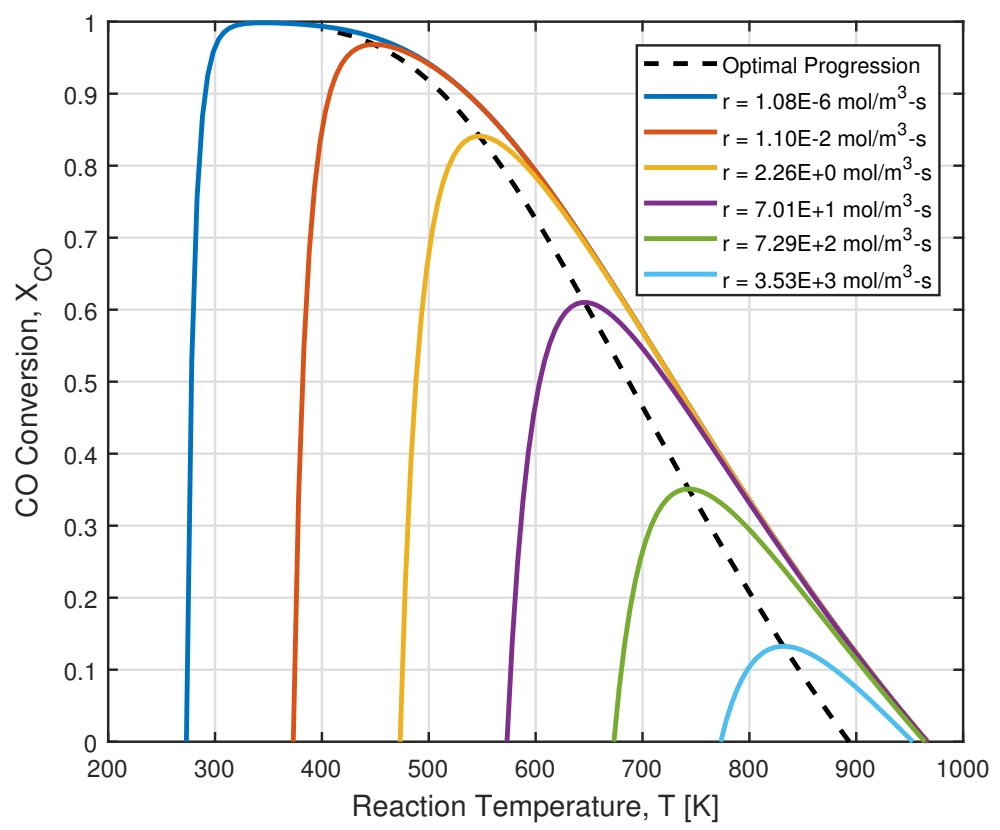

Fig. 2 Optimal progression of WGS reaction

a composition and temperature on the optimal reaction progression. A prescribed wall temperature condition following the optimal temperature profile was then applied for the remainder of the reaction channel length. An inlet temperature of $350^{\circ} \mathrm{C}$ and the same inlet reaction stream composition used for the isothermal model were used in this model.

\subsection{Heat Exchange Integrated Reactor Model}

To practically achieve a reaction temperature profile approaching the optimal temperature profile developed from Equation 10, a heat exchange integrated reactor model was developed by modifying the existing reactor model to include coolant channels. A "unit cell" of this reactor, consisting of half of a single reaction channel, half of a single coolant channel, and the stainless steel wall separating the channels, as shown in Figure 3, forms the basis of this model. The coolant channel has the same dimensions as the reaction channel and the wall separating the channels has a thickness of $0.6 \mathrm{~mm}$. The reactor operates in a counter flow configuration and air is used as the coolant. The inlet flow rate of cooling air is taken as $100 \mathrm{Nml} / \mathrm{min}$ and the inlet air temperature is taken as $257.25{ }^{\circ} \mathrm{C}$ to match the corresponding reaction channel outlet temperature from the prescribed optimal temperature model.

In addition to the transport phenomena modeled within the reaction channel using the equations listed in Section 2.1, the transport phenomena within the coolant channel and reactor wall are also modeled. The Navier-Stokes equations and energy conservation equations are again used to model the momentum transfer and energy transfer in the coolant channel, respectively. The subscript " $a$ " in these equations and the other transport equations listed is used to indicate air properties.

$$
\begin{gathered}
\rho_{a}(\mathbf{u} \cdot \nabla) \mathbf{u}=-\nabla P+\nabla \cdot\left(\mu_{a}\left(\nabla \mathbf{u}+(\nabla \mathbf{u})^{T}\right)-\frac{2}{3} \mu_{a}(\nabla \cdot \mathbf{u})\right) \\
\nabla \cdot\left(k_{a} \nabla T\right)-\nabla \cdot\left(\rho_{a} c_{p, a} \mathbf{u} T\right)=0
\end{gathered}
$$

The energy equation for the reactor wall only accounts for thermal conduction and simplifies to: 


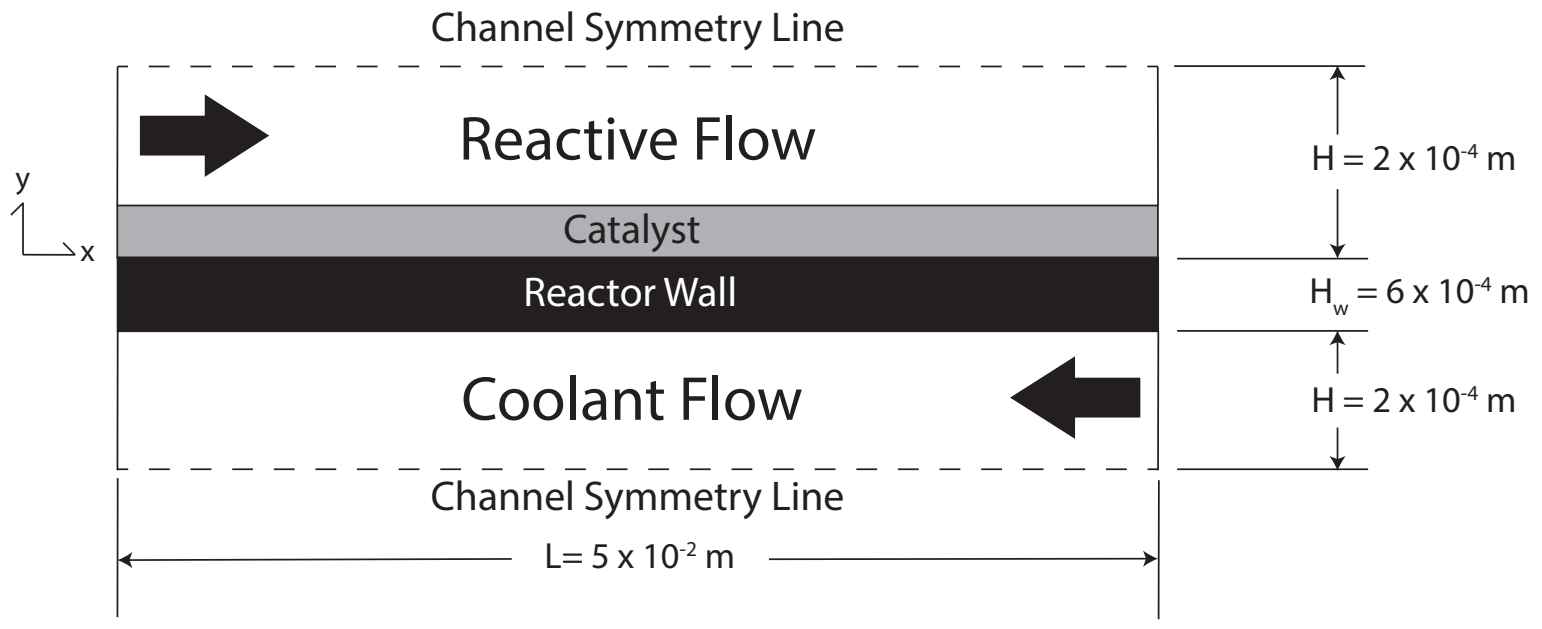

Fig. 3 2-D geometry of heat exchange integrated model

$$
\nabla \cdot\left(k_{w} \nabla T\right)=0
$$

The sensible energy increase of the coolant stream across the reactor can be determined using the following equation to evaluate the energy recovery performance of the reactor:

$$
q=\dot{M}_{a} c_{p, a}\left(T_{a, \text { out }}-T_{a, \text { in }}\right)
$$

\section{RESULTS AND DISCUSSION}

The isothermal model results are plotted with the experimental results generated by Germani and Schuurman [10] in Figure 4. The model results shown were taken as the average $\mathrm{CO}$ conversion value of the outlet of the reaction channel. Examining both trends, it can be seen that there is an initial increase in the reactor CO conversion as the temperature increases. At higher temperatures, the $\mathrm{CO}$ conversion begins to decrease as the reactor becomes thermodynamically limited as opposed to kinetically limited. Generally, the agreement between the numerical model and the experimental results is good, with the model accurately replicating the behavior of the experimental data over the entire range of temperatures examined. The largest percent error between the model and experimental results is $39.1 \%$ and occurs at a temperature of $493.15 \mathrm{~K}$. The absolute average percent error between the model and experimental results is $10.8 \%$. These results provide confidence that the analyses performed for the prescribed wall temperature profile and integrated cooling channel models are physically meaningful.

The results for the isothermal, prescribed wall temperature, and integrated coolant reaction channel models are shown in Figures 5, 6, and 7. Figure 6 shows the temperature profile versus reactor length for the three cases. Here, pure counterflow with air was assumed for the reactor with integrated channels, yielding an approximately linear drop in temperature for the reacting flow. Different flow arrangements, working fluids, etc., could be considered in an attempt to more closely approximate the optimal temperature profile. Figure 5 shows the $\mathrm{CO}$ conversion of both the prescribed temperature and integrated coolant models is significantly enhanced compared to the isothermal model. The isothermal model reaches a final equilibrium CO conversion of $75.5 \%$, but after a reactor length of approximately $0.015 \mathrm{~m}$, the reaction has reached $99 \%$ of this final conversion value. As a result, the majority of the reactor length is contributing relatively little to the overall $\mathrm{CO}$ conversion. The prescribed temperature model and the integrated coolant model are both able to further 


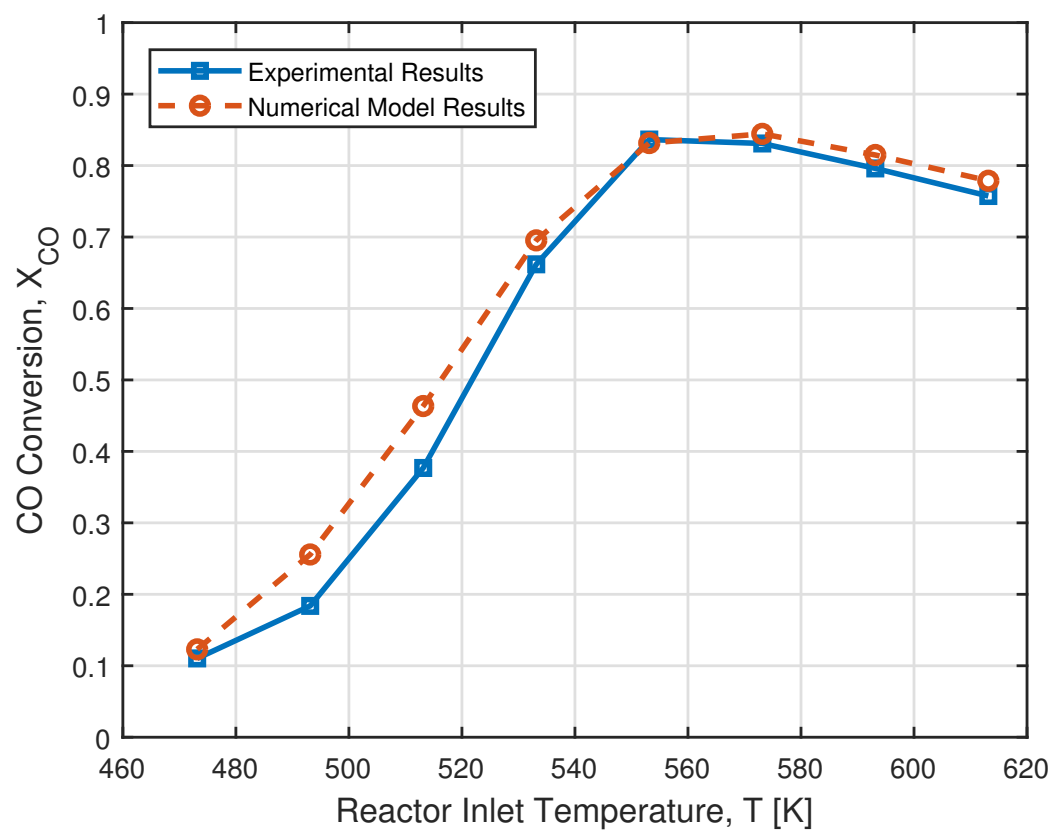

Fig. 4 Comparison of isothermal COMSOL model results with Germani and Schuurman [10] experimental results

enhance the $\mathrm{CO}$ conversion of the reactor over this remaining length by gradually cooling the reaction such that it becomes less thermodynamically limited.

Figure 7 shows the prescribed temperature model closely follows the optimal conversion profile developed from Equation 10 after operating isothermally initially. This results in the convex temperature profile shown in Figure 6 and a final $\mathrm{CO}$ conversion of $88.4 \%$. The integrated coolant model does not follow this temperature progression closely and instead takes on a linear distribution for the coolant flow rate and inlet temperature specified, but still achieves a CO conversion of $87.5 \%$, which is within a percent of the prescribed temperature value. This would indicate that for the conditions and geometry simulated, the deviation between the temperature profiles of the integrated coolant reactor and the prescribed temperature reactor minimally impacts the overall reactor performance.

Finally, a comparison of the reaction and coolant stream temperatures in the coolant integrated model is shown in Figure 8. For the conditions simulated, the reaction and coolant temperatures take on nearly identical linear distributions, with the reaction and coolant temperatures approaching values of $260.5^{\circ} \mathrm{C}$ and $347.5^{\circ} \mathrm{C}$ respectively. The modeled temperature increase of the air coolant is calculated to correspond to an energy increase of $7.48 \times 10^{-4} \mathrm{~W}$ using Equation 14 for a single channel. For a full reactor system producing hydrogen at an industrial scale of $1500 \mathrm{~kg}$ per day, this would scale up to a a total energy recovery of approximately $310 \mathrm{~kW}$ from the reaction stream which can potentially be used to useful effect somewhere else in the overall system.

\section{CONCLUSIONS}

This study explored the implementation of microreactors toward the process intensification of the WGS reaction. A numerical model developed in COMSOL Multiphysics was used to simulate the transport phenomena within a single microchannel of such a reactor. Upon validation of the model with experimental values found in the literature, the model was expanded to evaluate and compare reactor performance when it operates under isothermal, optimal temperature, and integrated cooling conditions. Preliminary results indicate that both the optimal temperature and integrated coolant reactor models achieve more than a $12 \%$ increase 


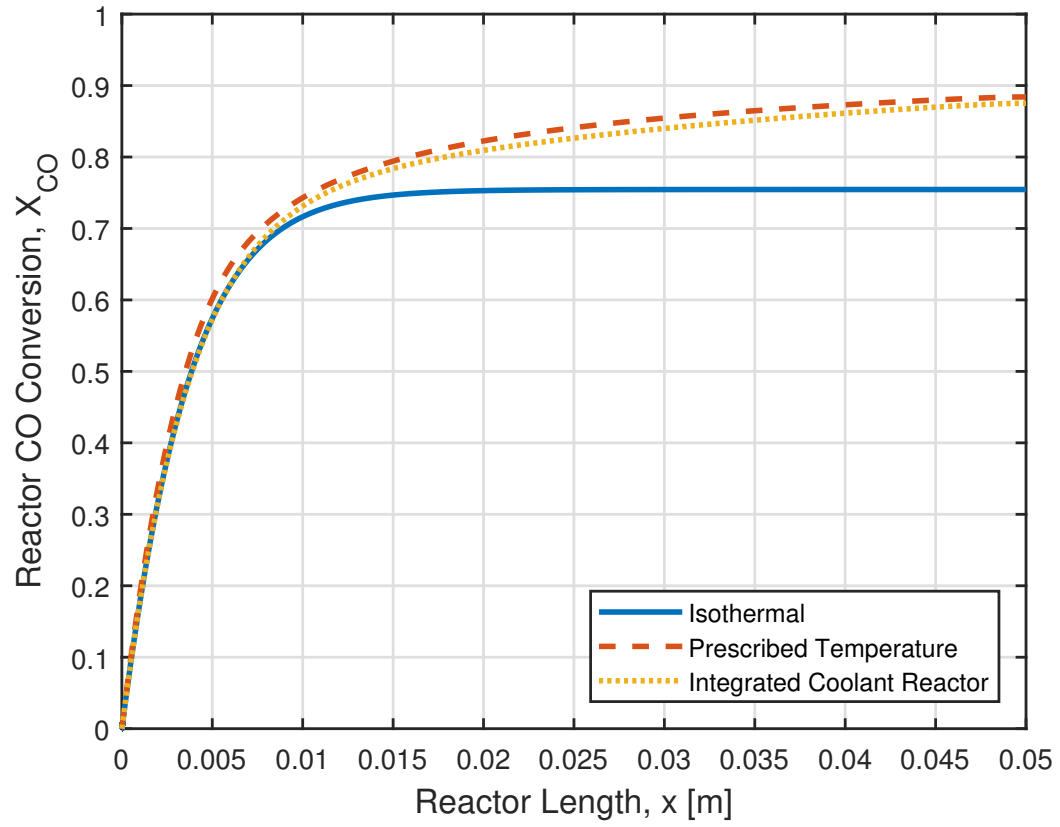

Fig. 5 Comparison of CO conversion profiles versus reactor length for each reactor model

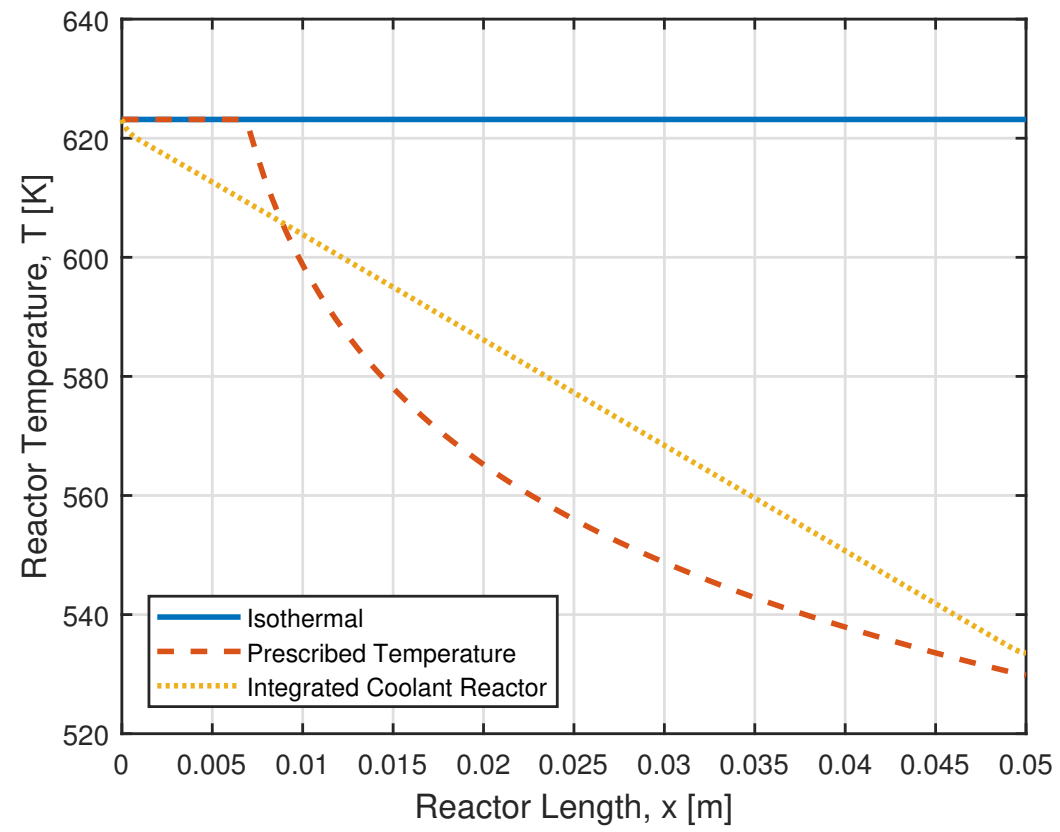

Fig. 6 Comparison of temperature profiles versus reactor length for each reactor model 


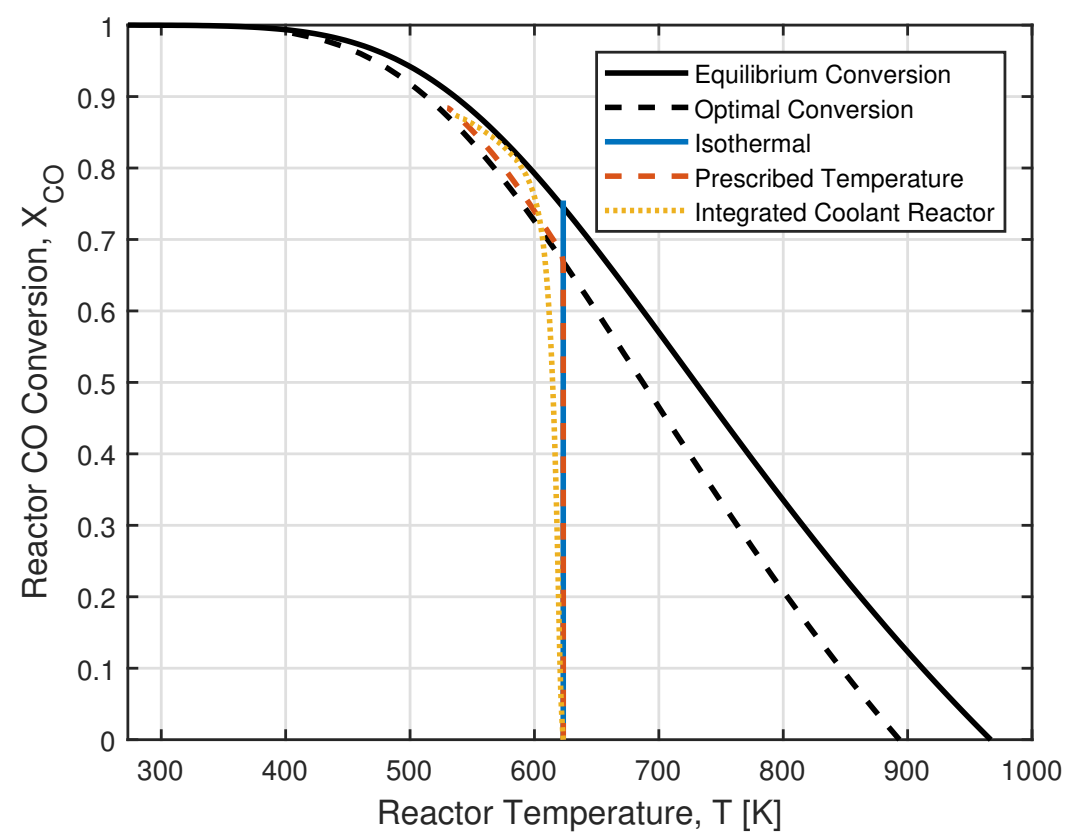

Fig. $7 \mathrm{CO}$ conversion plotted as a function of temperature for each reactor model

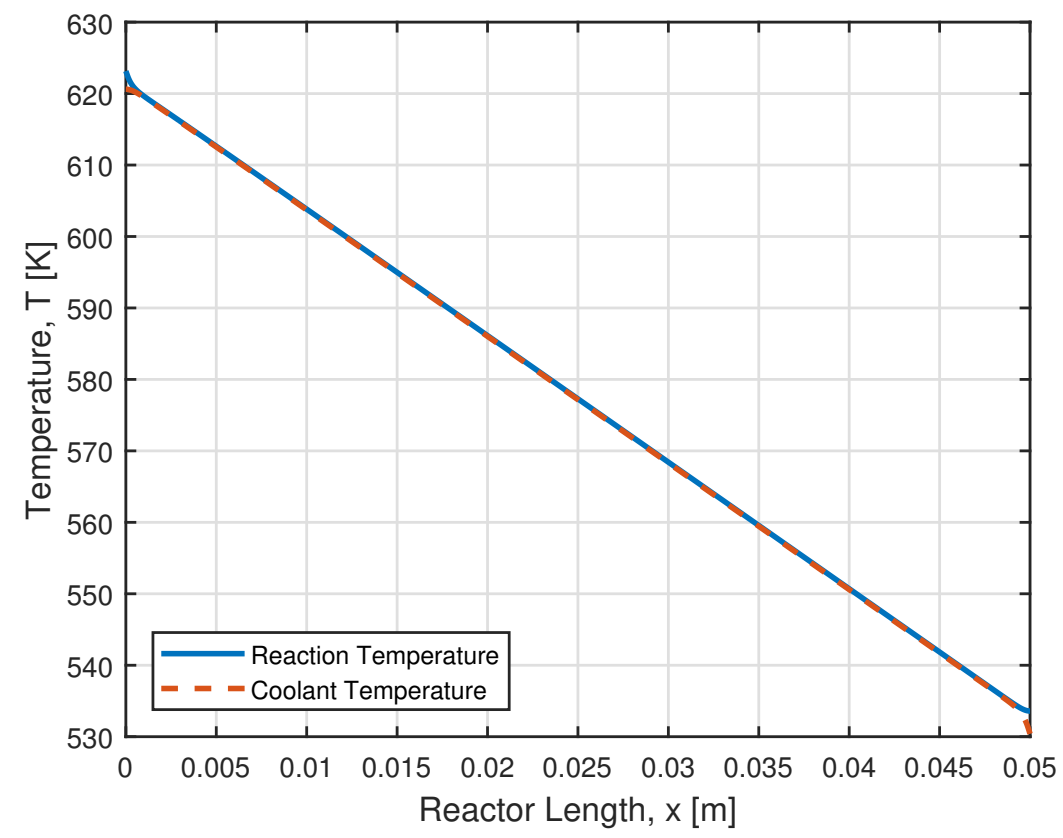

Fig. 8 Reaction and coolant temperatures in the heat exchange integrated model 
in CO conversion compared to the isothermal model for the inlet conditions and reactor architecture used. While the integrated coolant reactor does not closely follow the optimal temperature profile, it still achieves a $\mathrm{CO}$ conversion within a percent of the optimal temperature reactor, indicating nearly negligible performance degradation for the conditions and geometry simulated. This is not always the case, however, and it can prove beneficial in integrated cooling reactors to split the cooling into several stages to more closely replicate the optimal temperature profile. For the coolant integrated reactor, the coolant stream recovers $310 \mathrm{~kW}$ of sensible energy from the reaction stream at a production capacity $1500 \mathrm{~kg}$ per day. Such a recovery of energy from the reaction stream can be redirected to useful effect somewhere else in the overall steam reforming process. These preliminary results suggest that additional studies examining the optimization of WGS reactor performance in terms of its $\mathrm{CO}$ conversion and energy recovery in the context of the steam reforming process should be performed to determine the best reactor operating mode within an overall system.

\section{NOMENCLATURE}

$\begin{array}{lll}H_{c a t} & \text { Catalyst thickness } & (\mathrm{m}) \\ H_{W} & \text { Reactor wall thickness } & (\mathrm{m}) \\ \Delta H_{W G S} & \text { Heat of reaction } & (\mathrm{J} / \mathrm{mol}) \\ K_{e q} & \text { Equilibrium constant } & (-) \\ K_{i} & \text { Adsorption equilibrium constant of species } i & (1 / \mathrm{bar}) \\ k_{r d s} & \text { Reaction rate constant } & (\mathrm{mol} / \mathrm{kg}-\mathrm{s}) \\ q & \text { Reaction stream energy recovery } & (\mathrm{W}) \\ r & \text { Reaction rate } & \left(\mathrm{mol} / \mathrm{m}^{3}-\mathrm{s}\right) \\ W G S & \text { Water-gas shift } & (-) \\ x & \text { Axial position along reactor length } & (\mathrm{m}) \\ X_{i} & \text { Conversion of species } i & (-) \\ \epsilon & \text { Catalyst porosity } & (-) \\ \mathrm{\kappa} & \text { Catalyst permeability } & \left(\mathrm{m}^{2}\right)\end{array}$

\section{REFERENCES}

[1] E. Taibi, R. Miranda, W. Vanhoudt, T. Winkel, J.-C. Lanoix, and F. Barth, "Hydrogen from renewable power: Technology outlook for the energy transition," tech. rep., International Renewable Energy Agency, Abu Dhabi, 2018.

[2] C. Rhodes, G. J. Hutchings, and A. M. Ward, "Water-gas shift reaction: finding the mechanistic boundary," Catalysis Today, vol. 23, no. 1, pp. 43-58, 1995.

[3] Moe, "Design of water-gas shift reactors," Chemical Engineering Progress, vol. 58, no. 3, pp. 33-36, 1962.

[4] P. Kumar, E. Akpan, H. Ibrahim, A. Aboudheir, and R. Idem, "Kinetics and Reactor Modeling of a High Temperature WaterGas Shift Reaction (WGSR) for Hydrogen Production in a Packed Bed Tubular Reactor (PBTR)," Ind. Eng. Chem. Res, vol. 47, no. 12, pp. 4086-4097, 2008.

[5] C. Ratnasamy and J. P. Wagner, "Catalysis Reviews Water Gas Shift Catalysis Water Gas Shift Catalysis," Catalysis Reviews, vol. 51, no. 3, pp. 325-440, 2009.

[6] L. Kiwi-Minsker and A. Renken, "Microstructured reactors for catalytic reactions," Catalysis Today, vol. 110, pp. 2-14, 12 2005.

[7] S. Bac, S. Keskin, and A. K. Avci, "Modeling and simulation of water-gas shift in a heat exchange integrated microchannel converter," International Journal of Hydrogen Energy, vol. 43, pp. 1094-1104, 12018.

[8] E. L. Romero and B. A. Wilhite, "Enhancement of water-gas-shift conversion via externally imposed temperature profiles: Comparison of linear, convex and Gaussian profiles," Chemical Engineering Journal, vol. 175, pp. 433-442, 2011.

[9] W. E. Tegrotenhuis, D. L. King, K. P. Brooks, B. J. Golladay, and R. S. Wegeng, "10b Optimizing Microchannel Reactors by Trading-Off Equilibrium and Reaction Kinetics through Temperature Management," in 6th International Conference on Microreaction Technology, (New Orleans), pp. 1-11, 2002.

[10] G. Germani and Y. Schuurman, "Water-gas shift reaction kinetics over $\mu$-structured Pt/CeO2/A12O3 catalysts," AIChE Journal, vol. 52, pp. 1806-1813, 52006. 